\title{
BMJ Open Quality Acute hospital preoperative assessment redesign: streamlining the patient pathway and reducing on-the-day surgery cancellations
}

\author{
Debbie Brazil (D) , ${ }^{1}$ Charlotte Moss, ${ }^{2}$ Karen Blinko ${ }^{3}$
}

To cite: Brazil D, Moss C, Blinko K. Acute hospital preoperative assessment redesign: streamlining the patient pathway and reducing on-the-day surgery cancellations. BMJ Open Quality 2021;10:e01338. doi:10.1136/ bmjoq-2021-001338

- Additional supplemental material is published online only. To view, please visit the journal online (http://dx.doi.org/10. 1136/bmjoq-2021-001338).

Received 5 January 2021 Accepted 14 August 2021

\section{D) Check for updates}

(C) Author(s) (or their employer(s)) 2021. Re-use permitted under CC BY-NC. No commercial re-use. See rights and permissions. Published by BMJ.

${ }^{1}$ Integration and Transformation Team, Bedfordshire Hospitals NHS Foundation Trust, Luton, UK ${ }^{2}$ Anaesthetics, Bedfordshire Hospitals NHS Foundation Trust, Luton, UK

${ }^{3}$ Pre-Operative Assessment, Bedfordshire Hospitals NHS Foundation Trust, Luton, UK

Correspondence to

Debbie Brazil;

debbie.brazil1@nhs.net

\section{ABSTRACT}

Introduction Avoidable surgery cancellations in an acute trust were often attributed to inadequate preoperative assessment. These assessments, undertaken shortly before surgery, were delivered across eight different locations, $60 \%$ by a central nursing team and the remainder by other healthcare professionals. There was inconsistency in what and who were assessed, and inadequate time to optimise patients. There was difficulty finding capacity for urgent patient assessment, plus a lack of a pool of 'ready-assessed' patients to fill last-minute operating list gaps.

Methods A diagnostic phase using data analysis, root cause analyses and clinic observations identified multiple systemic issues confirming the need for system change. Interventions 0ther trusts operating different models were visited and their processes were adapted to create a preassessment model relevant to our trust context. Key features included early preassessment, triage and streaming, in-clinic support from a prescribing pharmacist and consultant anaesthetist, a standardised outcome form documenting specific patient requirements needing action when a surgery date was agreed, surgery dating only on confirmation of patient optimisation, an administrative office (hub) with a tracking database to coordinate follow-up tasks and a patient hotline. A key enabler was a single, bespoke location. Where possible, testing took place in advance of the go-live. However, due to the transformational nature of the new model, some changes could only be tested and refined at scale in the new, single location.

Results Two months post implementation, a preliminary audit was positive, but clinic observations indicated that patient clinic flow was suboptimal. Further structural and process modifications were made. Ten months post implementation, a further root cause audit showed a near-elimination of on-the-day surgery cancellations for patients assessed in the redesigned service.

Conclusion The bundle of 17 interlinked interventions proved highly effective in delivering sustained improvements, which could be adopted by other trusts.

\section{PROBLEM}

Our 650-bedded District General Hospital provides surgical services over multiple specialties, including tertiary head and neck, and bariatric care. Approximately 18000 adult patients are added annually to the elective surgical waiting list, with $80 \%$ requiring preoperative assessment to ensure preparation and optimisation ahead of sedation or general anaesthetic procedures. ${ }^{1}$

Of all booked elective procedures, $7 \%$ (November 2015-May 2017, average 170 pm) resulted in 'on-the-day' surgery cancellations, and there was a prevailing view that a large percentage were driven by inadequate preoperative assessment processes. Three months of cases were audited (August-October 2015) where the cancellation reason recorded was patient unfit, unwell on the day or operation declined (118 total, $39 \mathrm{pm}, 23 \%$ ). Of these, $69 \%$ (81) were found to be avoidable or potentially avoidable with $73 \%$ of those, with systemic root causes, being within the preassessment phase.

Additional problems cited by administrative staff included lack of capacity to assess clinically urgent patients at short notice and lack of a pool of ready-assessed patients to fill last-minute gaps on operating lists, contributing further to underused theatre lists.

An in-depth diagnostic process highlighted multiple problems contributing to on-the-day surgery cancellations:

- Paucity of documented policies including no formal eligibility criteria, and therefore inconsistencies in who received preassessment.

- Assessments delivered by nine groups of staff in eight inadequate, geographically distanced locations. The central preassessment nurse team delivered $60 \%$, with the remainder delivered by other specialty staff or teams, including some without specific training.

- A range of preassessment documentation templates were used by the different groups of staff, and the surgery booking team was notified of assessment outcomes 
in different ways, increasing the risk of omissions and errors.

- Typically, a preassessment appointment was booked close to the booked surgery date, leaving negligible time for optimisation, even when the original addition to the waiting list had been several weeks or months before.

- During assessments, nurses frequently had to find an available doctor to obtain a required prescription, which was unreliable and time-consuming.

- Nurses were expected to follow up results for their individual patient case load. However, with no allocated administrative time, this process was unreliable and issues were sometimes missed. Handover of outstanding issues when staff took leave relied on goodwill.

- Where indicated, nurse queries were escalated to a patient's allocated procedural anaesthetist for review, and notes went physically back and forth between the preassessment area and the anaesthetic office. Anaesthetists were often unable to conduct timely reviews, and nurses lacked time to follow up requested actions.

- There was no process for ensuring that any surgery date that was rescheduled after the preassessment had taken place had any identified presurgery dependencies reprovided, such as revised date for cessation of medication. Patients were therefore not optimised on the day of surgery.

- On the day of surgery admission, poorly prepared patients were identified and outstanding activities were urgently arranged, creating unquantified noise in the system and staff stress. Operating lists were delayed, while lists were adjusted to accommodate last-minute changes, but sometimes the last scheduled patient 'timed out'.

Cumulatively, these issues were inconvenient and distressing for patients and staff, contributing to longer waits for all patients, with corresponding impact on the 18-week waiting time target. It also contributed to a loss of productivity and income for the trust, and retention and recruitment difficulties in the preassessment team because of the difficult environment and low morale.

The aim of the redesign project was to therefore address these drivers and to eliminate avoidable lastminute cancellations.

\section{BACKGROUND}

Nurse-led, multidisciplinary, preoperative assessment has become accepted practice, providing the opportunity to optimise treatment of existing disease, making a detailed plan for care during and after surgery, reducing patient anxiety levels and surgery cancellations, and supporting efficiency of operating lists. There should be anaesthetic reviews of medical notes for patients with increased risks of mortality and morbidity, with face-toface consultations for the highest-risk patients to inform the consent process. Service design features should include preoperative assessment taking place as early as possible in the patient's surgical pathway; it should ideally be one-stop so that patients can attend during the same hospital visit as their surgical outpatient appointment, and it should have pharmacy input to support patients with polypharmacy. ${ }^{1-3}$

Risk stratification is also being used increasingly, where patients have an initial assessment and are then streamed for further assessment according to clinical complexity. This improves in-clinic productivity and patient experience. Enablers are structured triage, separation of administrative and clinical tasks, and dedicated anaesthetic resource. ${ }^{34}$

Centralised models are increasingly common, driven by the complexities of modern medicine, with multiple medical and non-medical specialists involved in decision making. The former, more traditional, linear process is increasingly unable to deal with this. Centralisation also enables previously unfeasible, logistically difficult, underused preoperative interventions, such as one-stops, triage and perioperative pharmacy, to be economically incorporated. ${ }^{3}$

Even with centralisation, there are challenges in having patients drop in to preoperative assessment direct from outpatient clinics, with peaks arising which correspond to peaks in outpatient clinics. These need to be carefully managed alongside any booked preassessment patient appointments. ${ }^{5}$

The importance of internal onward communication after preassessment, of essential information to the right staff, is poorly understood and is also not linear. The fragmented multidisciplinary nature of preassessment results in information accumulating as the patient flows between nurses, pharmacists, physicians, administrators and surgeons. Unfortunately, failures of information transfer and communication errors occur sometimes due to the use of multiple information technology systems used by these different staff groups, which are not discrete in that information lost early on is sustained thereafter and can potentially compromise safety downstream or lead to on-the-day surgery cancellations. Few organisations have developed a systematic approach to address this. ${ }^{3}$ Our process mapping and initial audit found that rescheduling surgery dates was the greatest point for this risk and it was a revelation.

The ideal preoperative model should always be adapted to local circumstances, drawing out common controversial issues to be resolved in each local institution. This may include achieving the right balance between clinical protocols and clinical freedom, or achieving adequate levels of trust in the system, ${ }^{6}$ such as gaining agreement from procedural anaesthetists to accept the assessment and preparation of a patient by a preassessment anaesthetist colleague.

\section{MEASUREMENT}

We took a four-pronged approach to establish the current state (online supplemental file 1, diagnostic phase). 
1. Specialty information was gathered, including which patient cohorts were preassessed; nurse staffing resource; location; and what the preassessment covered, including generic versus specialty-specific elements. Anaesthetist resource and workload were also assessed using a prospective audit.

2. Baseline trends for surgical activity and on-the-day surgery cancellations were reviewed, drawn from the patient administration system (PAS).

3. Central preassessment clinic observations took place confirming patient, staff and information flows, as well as recording unconscious empirical behaviours. Selective process mapping was conducted and was pivotal in revealing the communications and information flow disconnect, which explained a range of apparently unrelated cancellations.

4. Anaesthetists and an improvement lead conducted root cause analysis of assumed preassessment-related cancellations for August-October 2015. Each cancellation was categorised for avoidability, pathway stage and whether the cause was a 'system' or 'one-off human error'. Lost income opportunity was estimated, pro rata, at $£ 400 \mathrm{k}$ pa from the $69 \%$ of avoidable or potentially avoidable cancellations. There were 39 different avoidable root causes found, of which 27 were systemic with potential for resolution. Seventy-three per cent of these occurred at the preassessment stage, or after this but prior to surgery date (online supplemental file 2).

\section{DESIGN}

The project scope was confirmed: to redesign the elective preoperative pathway period, from the time a patient was added to the elective surgery waiting list to the day of surgery.

The redesigned model (online supplemental file 3) required a bundle of interlinked changes to be implemented, which fundamentally transformed the hospital's preoperative system and processes, and when delivered together were expected to significantly reduce avoidable on-the-day surgery cancellations (online supplemental file 1 , design phase including timeline). They included the following:

- Agreeing a formalised policy defining requirement and eligibility for preassessment, summarised as

- Patients having procedures requiring an anaesthetist (eg, general anaesthetic, regional block and sedation).

- Local anaesthetic procedures at the request of the surgeon (eg, requiring medication advice).

- Patients would be offered preassessment as soon as they were listed for surgery (rather than 1-2 weeks before the surgery date) with a choice of 'drop-in' from the outpatient clinic or during the following days, giving maximum time for preparation and optimisation.

- A bespoke unit was built specifically for the preassessment service. Running from a single location meant working hours could be extended more economically, opening between 08:00 and 18:00, and facilitated the drop-in service.

- Patients who did not 'drop in' within 2 weeks would be posted a prebooked appointment. If they did not attend this, capacity was simply used on the day for drop-in patients, reducing waste.

- The drop-in model would mean that there was always capacity to prioritise urgent patients.

- Dedicated preassessment nurses would staff the unit, meaning specialty teams formerly delivering preassessment to their specialty patients could be redeployed to specialty work. Exceptions were ophthalmology and complex head and neck surgery.

- A prescribing pharmacist was introduced to review appropriate patients and to provide a prescribing service, funded for half a day as a cost/benefit compromise.

- Consultant anaesthetists had existing establishment funding extended to the equivalent of half of every day, to see high-risk patients face to face, perform notes reviews and support nursing staff. A cohort of specific anaesthetists would deliver this, to increase consistency of decision making and because the procedural anaesthetist was unknown at the point in time that a patient was added to the elective surgery waiting list. Communication of critical information to the procedural anaesthetist would be facilitated using newly established communication processes.

- A new triage and streaming process was introduced, facilitated by the single location, using a newly designed and tested standardised triage tool. This would be delivered by healthcare assistants (HCAs) and supervised by registered nurses. Low-risk patients would be triaged home and 'green-lighted' for surgery dating. More complex patients were streamed to an onward appointment with one or more of a pharmacist, nurse and anaesthetist. Historic American Society of Anaesthesiologists (ASA) status patient data suggested that $20 \%$ of patients would no longer need to see a registered nurse, but the HCA workforce would need to increase. This would enable the central team to absorb patients previously not assessed at all or assessed by specialty teams. The triage tool would replace the original preassessment booklet, and the theatre's medical record booklet would be aligned in parallel. This would improve overall use of resources as well as improve patient experience.

- Patients streamed to see a nurse would be one-stopped whenever possible rather than be asked to return another day.

- Administrative follow-up would be delivered by a rostered HCA and registered nurse in a colocated preassessment 'hub', which would carry out the following functions:

- Ongoing follow-up of diagnostic tests.

- Tracking patients whose preassessments had outstanding actions to a conclusion. A bespoke 
tracking database tool was developed to support this, with daily worklist reports.

- Telephone 'hotline' for patient queries.

- Liaison across anaesthetic, surgical and surgery booking office teams.

- Preassessments would be valid for 3 months unless notified of a change by a patient. In the situation where they 'timed out', the hub nurse would conduct a telephone follow-up according to a proforma to assess changes and address required actions.

- A standardised spreadsheet 'outcome' form was introduced to communicate to the surgery booking office patients' suitability for surgery dating and any dependent actions required when the patient was allocated a date for surgery, such as cessation of medication. This was later incorporated into the database tracking tool and the surgery booking team was given read access. This key, shared, management and communication tool became another key enabler.

- Green-lighted patients would create a pool of 'readyto-go' patients who could be booked at short notice to use last-minute theatre capacity.

- Once the service was working at steady state, a suite of key performance indicators were developed for management of demand and capacity to track the activity and efficiency of the service.

\section{STRATEGY}

Our redesign followed a general quality improvement philosophy such as that promoted by NHS Improvement's Quality Service Improvement Redesign, and drawing broadly on the philosophies of Lean and Model for Improvement and using individual tools as appropriate. ${ }^{7-9}$ It resulted in a complex system transformation across the surgical patient pathway, involving implementation of multiple changes to address multiple drivers, where resolving any one alone would not make a measureable difference. Small tests of change could generally only be applied where an individual change was self-contained. Where solutions were larger and more complex, requiring infrastructure change, the Plan Do Study Act (PDSA) principle of measure, test and iteration was followed but conducted post-go-live. Reed and Card recognise that in the real world, one size does not fit all. ${ }^{10}$

The technical redesign would not have been enough, or as successful alone, if the conditions for change and the right leadership had not also been in place at this particular time. Dixon-Woods notes that this is a key reason why the exact same changes applied and adapted locally elsewhere may still not replicate as successfully. ${ }^{11}$

The project was led by an improvement lead who coordinated the project's six workstreams, in partnership with the lead preassessment nurse and anaesthetist. Membership was drawn from internal stakeholders, including anaesthetists, managers, nursing and surgery booking staff. The workstreams worked on the finer details of the pathway design. Other hospitals were visited or consulted remotely to provide them with ideas and inspiration. The proposed new model underwent wider consultation with all relevant staff, including anaesthetists, surgeons and nursing staff, and formed the basis of the business case.

While waiting 1 year for the new location to be repurposed, the work of introducing and testing individual change ideas took place wherever possible, to facilitate the transition (online supplemental file 1, transition period), including

- The standardised outcome form, which was introduced with staff and the booking team and then iteratively improved.

- The standardised triage tool, which was iteratively tested and refined with patients using the existing preassessment paperwork as a control for safety.

- A paper format version of the tracking tool was introduced, allowing staff to familiarise themselves with the concept and highlighting the advantages, while the database version was developed and deployed. The full benefits were only realised in the new single location.

- A major Methicillin-resistant Staphylococcus aureus (MRSA) swabbing project. Mindful that assessing patients much earlier in their surgical journey would mean MRSA swabs would no longer be valid (validity 28 days), a parallel 9-month project was undertaken to quantify and evaluate the impact for our specific local population. This was in the context of discursive rather than directive MRSA national guidance from the UK devolved health administrations. ${ }^{12-14}$ It demonstrated that those deemed low risk, who tested negative initially, were unlikely to become positive during the wait for surgery even where that was 6 months. The infection control team agreed to update trust guidance, increasing the validity of a negative swab result to 6 months. Exceptions were those in higherrisk categories who would need to return for further swabbing, which would be tracked on our database.

A 6 month transition period of double-running clinics was required, which started 3 months before the new model was introduced, needing temporary additional staff, to clear the legacy patients already waiting for surgery without a date and no preassessment.

Each surgical specialty was transitioned into the new model, in turn, over 3 months from September to November 2018 to allow for individual surgeon training and to allow familiarisation with the process from the outpatient nursing perspective. This ensured that the onward sign posting of patients from outpatients to preassessment was successful. It also allowed preassessment staff time to adjust to the new way of working.

\section{PATIENT AND PUBLIC INVOLVEMENT}

This project was developed without direct patient involvement in the overall design, but with input in targeted areas, such as paperwork development. One of the project aims was to address what was evidently 
poor patient experience. A patient survey was introduced with patients in the centralised clinic to provide continuous qualitative feedback. Analysis from the first 2 months' data showed that the drop-in service was overwhelmingly positive but identified concerns about in-clinic waiting times. Changes allowed patients to wait or book a preferred date to return, alongside the introduction of a flow manager, and resulted in improvements, with the queue self-regulating.

\section{RESULTS}

After go-live was complete, there were three formal points of measurement that led to modifications and retesting.

A 2-month post implementation repeat root cause audit (January 2019 data) demonstrated that avoidable cancellations were down from $69 \%$ to $52 \%$ due to a reduction in central preassessment team related cancellations and better communications with anaesthetists. However, it highlighted that targeted individual staff training was still needed. Further audit was postponed due to the presence of a large number of legacy patients, with old issues inevitably recurring.

Repeat clinic observations took place and identified that patients appreciated the one-stop offer. However, the shift from prebookable to drop-in appointments was suboptimal with some drop-in patients waiting over 2 hours. This was also evident from our patient survey. We therefore introduced the option for patients to return for a booked appointment of their choice, after which the queue self-regulated, and this was well received.

A further audit 10-month post implementation (August-October 2019 data) included only patients from specialties preassessed within the new model, so the 2015 audit results were recalculated to be comparable. This final audit demonstrated a reduction in avoidable preassessment cancellations as a percentage of all elective cancellations from a recalculated figure of $8 \%$ (23) in August-October 2015 to 1.2\% (4) in August-October 2019, which was outside of the 2015 confidence limits and which can be concluded was likely to be the result of the redesigned system.

The activity and income trend shows the step change in preassessment activity and income after implementation (online supplemental file 4).

All other anticipated benefits were delivered and were in some cases better than expected; for example, $74 \%$ of patients were triaged after HCA assessment for nurse assessment rather than the estimated $80 \%$, and productivity was higher than the business case modelling.

\section{LESSONS AND LIMITATIONS}

It has proven difficult to measure and quantify the full impact of the transformation. The PAS on-the-day surgery cancellation data were unsuitable to use to track preassessment-related cancellations because PAS data did not accurately capture the root cause reason or avoidability of these cancellations. Using all surgery cancellations as a proxy measure was unsuitable because it is heavily impacted by seasonality and because preassessment-related cancellations are a small overall percentage. Manually auditing the root causes of cancellations is resource intensive and unsustainable to do routinely. Therefore, it has not been possible to robustly confirm the reduction in preassessment-related cancellations using statistical process control analysis.

Our measurement has also focused primarily on the 'catastrophic failure' of surgery cancellations. It would have been useful to audit other measures, such as the procedural anaesthetists' views of whether the preparation of patients for surgery had improved, but again this would require a non-sustainable bespoke data collection.

The principle of failing quickly with small tests of change was not always possible because some change ideas were dependent on investing in the new single location and a modest increase in the HCA workforce. However, the rigorous evidence base from the diagnostic audit gave confidence that the risks were low.

Factors in the project's success included having organisation-wide support because the perceived failures of preassessment had reached a tipping point of organisational frustration. It also benefited from a strong senior sponsor providing visible oversight and commitment, a strong redesign leadership team made up of an experienced improvement lead, a preassessment nurse lead and a consultant anaesthetist lead. They shared the vision, each bringing a complementary skillset that was crucial to getting the design right, as well as in securing engagement from colleagues. The redesign work evolved into a programme of work with a large number of interlinking projects addressing the different drivers. A dedicated change lead meant that the project had the resource to coordinate and deliver this. If any one of these elements were missing, it would have been less successful or unsuccessful.

Having time at each stage of the project ensured that the drivers were properly diagnosed and allowed an integrated set of solutions to emerge with a slow burn implementation spanning more than a year. Key enablers were identified and implemented, including the single location, and the development of the hub tracking database. The latter was essential to streamline hub casework, provide daily worklists and mitigate the risks that would have been present in a paper-based system. Its migration into a communication tool was also pivotal.

The benefits of visibly following a structured process of improvement and continual reflective practice and trial and error have been key to service refinement, and have built staff trust and engagement. All of this has contributed to ownership and sustainability from the staff delivering the service and those working with them.

In hindsight, the double-running transition period should have started earlier as it overlapped with go-live. 
Resistance to centralisation was anticipated, especially in specialties where preassessment had been delivered historically by a lone nurse, but taking a criteria-based approach mitigated some of the issues. Two specialties withdrew from the new model soon after go-live, one when differences could not be reconciled, and the other when it emerged that their requirements were uniquely different. The latter have benefited by gaining a better understanding of their needs, which they can now address. With the evident success of the new model, one self-excluded service initiated talks to transfer their preassessment to the new centralised service.

A continuing concern was the number of non-urgent patients who were still being preassessed near to the date of their surgery, resulting in all the old problems. Further review highlighted that a significant number were added to the surgery waiting list outside of outpatient clinics. A new weekly report identifying patients not attending preassessment within 2 weeks of being added to the waiting list was introduced so the booking team could send them a prebooked appointment at that point, thereby mitigating this issue.

Bringing half a day of a pharmacist and anaesthetist into the clinic has been key to creating an integrated and timely assessment process, and we feel that patients would benefit from a full-day pharmacist, but the cost is a barrier.

While the success of this has been due to all identified solutions being implemented as a bundle, partial implementation would still benefit preassessment services, for example, clear eligibility criteria, a standardised outcome form, a triage and streaming process, access to a pharmacist and a cohort of preassessment anaesthetists.

\section{CONCLUSION}

The project has been seen as a major success within the trust, delivering all expected benefits. The risk stratified triage model, delivered from a single location, enabled the central team to preassess more patients than previously. This included those previously delivered by a number of other specialties and a cohort of patients who were not being preassessed at all, with only a small increase in HCA resource. In addition, the preassessment was to a higher standard and consistent.

The strength of understanding gained from the diagnostic phase meant that we implemented the right solutions at the outset. Our innovative package of interventions took the best ideas observed elsewhere and adapted them to our local context, enriched by our own ideas in a combination that we believe is unique.

We now deliver a modern, patient-centred preoperative assessment service, in line with published research, and it is an example of a robust implementation of Royal College Of Anaesthetist guidelines. The changes are fully embedded and the new model is sustained, with the preassessment team continuing to innovate.
Following the merger with a neighbouring trust in April 2020, they have requested to adapt and adopt this preassessment model.

Much can be copied by other organisations interested in improving their preassessment service, but it is recommended that they start by understanding their specific local context to assess which elements will work for them, and follow the principles of a quality improvement process.

Acknowledgements We thank colleagues at the Luton \& Dunstable Hospital, including the Surgery Division, which helped develop the model and engage colleagues; Flavia Menezes, who provided clinical advice for the first root cause analysis audit; and staff from the central preoperative assessment team, which helped shape, test and implement the change ideas, and embraced the new ways of working with such enthusiasm. We also thank the preassessment staff at Sheffield Teaching Hospitals, The Lister and Manchester Royal Infirmary for sharing their own innovative ways of working with us.

Contributors DB: primary author, quality improvement advice, initial concept, project design, data collection and analyses, project management of the implementation and write-up; CM: secondary author, anaesthetic advice, project design, implementation team and drafted the manuscript; KB: preassessment nurse advice, project design and implementation team.

Funding The publication of this paper is being funded by the Health Foundation- $Q$ Community.

Competing interests None declared.

Patient consent for publication Not required.

Provenance and peer review Not commissioned; externally peer reviewed.

Data availability statement Data are available upon request.

Supplemental material This content has been supplied by the author(s). It has not been vetted by BMJ Publishing Group Limited (BMJ) and may not have been peer-reviewed. Any opinions or recommendations discussed are solely those of the author(s) and are not endorsed by BMJ. BMJ disclaims all liability and responsibility arising from any reliance placed on the content. Where the content includes any translated material, BMJ does not warrant the accuracy and reliability of the translations (including but not limited to local regulations, clinical guidelines, terminology, drug names and drug dosages), and is not responsible for any error and/or omissions arising from translation and adaptation or otherwise.

Open access This is an open access article distributed in accordance with the Creative Commons Attribution Non Commercial (CC BY-NC 4.0) license, which permits others to distribute, remix, adapt, build upon this work non-commercially, and license their derivative works on different terms, provided the original work is properly cited, appropriate credit is given, any changes made indicated, and the use is non-commercial. See: http://creativecommons.org/licenses/by-nc/4.0/.

ORCID iD

Debbie Brazil http://orcid.org/0000-0003-2015-339X

\section{REFERENCES}

1 Royal College of Anaesthetists. Guidelines for the Provision of Anaesthesia Services for Preoperative Assessment and Preparation [Internet], 2019. Available: https://www.rcoa.ac.uk/gpas/chapter-2

2 Hines S, Munday J, Kynoch K. Effectiveness of nurse-led preoperative assessment services for elective surgery: a systematic review update. JBI Database System Rev Implement Rep 2015;13:279-317.

3 Kalamas A. Preoperative evaluation and management. In: Kaye A, Fox III C, Urman R, eds. Operating room leadership and management. 2nd edn. Cambridge Medicine, 2019: 173-84.

4 Monitor. Helping NHS providers improve productivity in elective care. England, 2015: Oct Publication code: IRRES 15/15. https://assets. publishing.service.gov.uk/government/uploads/system/uploads/ attachment_data/file/466895/Elective_care_main_document_final.pdf

5 Stark C, Gent A, Kirkland L. Improving patient flow in pre-operative assessment. BMJ Qual Improv Rep 2015;4. doi:10.1136/bmjquality. u201341.w1226. [Epub ahead of print: 0701 2015]. 
6 Anesthesia Key [Internet], Kerridge R. Changing the preoperative process a review of the evidence, 2017. Available: https://aneskey. $\mathrm{com} /$ changing-the-preoperative-process-a-review-of-the-evidenceross-kerridge/

7 Health Foundation. Quality improvement made simple What everyone should know about health care quality improvement [Internet]. 3rd edn, 2021. https://www.health.org.uk/sites/default/files/Qualitylmpro vementMadeSimple.pdf

8 NHS Improvement. Quality, Service Improvement and Redesign [Internet], 2017. Available: https://www.england.nhs.uk/wp-content/ uploads/2020/08/QSIR-A5-4pp.pdf

9 Parry G. Lean and IHI's Approach to QI: Do You Have to Choose? [Internet] Institute for Healthcare Improvement, 2016. Available: http:// www.ihi.org/communities/blogs/lean-and-ihi-s-approach-to-qi-doyou-have-to-choose\#: :text=Both\%20approaches $\% 20$ concentrate $\%$ 20on\%20the,decide\%20what\%20to\%20do\%20next

10 Reed JE, Card AJ. The problem with Plan-Do-Study-Act cycles. BMJ Qual Saf 2016;25:147-52.
11 Dixon-Woods M. Perspectives on context: the problem of context in quality improvement, Health Foundation, 2014. Available: https:// www.health.org.uk/sites/default/files/PerspectivesOnContextDix onWoodsTheProblemOfContextInQualitylmprovement.pdf

12 Hussey R, White J. MRSA screening letter, Welsh government, 2013. Available: http://www.primarycareservices.wales.nhs.uk/ sitesplus/documents/1150/CMO\%202013\%204\%20CNO\% 202013\%202.pdf

13 Department of Health. Implementation of modified admission MRSA screening guidance for NHS (2014) prepared by ARHAI MRSA screening implementation group. Available: https:// assets.publishing.service.gov.uk/government/uploads/system/ uploads/attachment_data/file/345144/Implementation_of modified_admission_MRSA_screening_guidance_for_NHS.pdf

14 Health Protection Scotland. Protocol for CRA MRSA screening national Rollout in Scotland, 2018. Available: https://www.hps. scot.nhs.uk/resourcedocument.aspx? resourceid=1639 